\title{
Rheo-Optical and Dielectric Study on Dynamics of Bottlebrush-Like Polymacromonomer Consisting of Polyisoprene Main Chain and Polystyrene Side Chains.
}

Hiroshi Iwawaki ${ }^{\dagger}$, Osamu Urakawa ${ }^{\dagger}$, Tadashi Inoue ${ }^{*} \dagger$, Yo Nakamura ${ }^{\ddagger}$, Yumi Matsumiya,॥ and Hiroshi Watanabell

'Department of Macromolecular Science, Graduate School of Science, Osaka University, 1-1 Machikaneyama-cho, Toyonaka, Osaka 560-0043, Japan

Department of Polymer Chemistry, Graduate School of Engineering, Kyoto University, Katsura, Nishikyoku, Kyoto 615-8510, Japan

"Institute for Chemical Research, Kyoto University, Uji, Kyoto 611-0011, Japan

*Corresponding author. Email: tadashi@chem.sci.osaka-u.ac.jp 
Figures S1-S3 show raw data of $\varepsilon^{\prime}$ and $\varepsilon^{\prime \prime}$ measured for the PI-PS-bPM/A300 sample at 120, 130, and $140^{\circ} \mathrm{C}$, respectively. A direct current (dc) conduction (due to ionic impurities) contributes to the $\varepsilon^{\prime \prime}$ data at low $\omega$, in particular at high $T$. This contribution (black lines), $\sigma / \omega$ with $\sigma$ being the dc conductivity, was subtracted from the $\varepsilon^{\prime \prime}$ data to evaluate $\varepsilon^{\prime \prime}$ of the PI-PS-bPM/A300 sample attributable to the end-to-end relaxation of the PI backbone. The plots of raw $\varepsilon^{\prime \prime}$ data already show a shoulder corresponding to this relaxation, so that the subtraction can be made with an acceptably small uncertainty (less than $20 \%$ uncertainty of the subtraction for the filled red squares in Figures S1-S3).

A comment needs to be made for the $\varepsilon^{\prime}$ data. The $\varepsilon$ data include the same information as the $\varepsilon^{\prime \prime}$ data, and a derivative $-\{\pi / 2\} \partial \varepsilon / \partial \ln \omega$ can be utilized for analyzing this information if changes of $\varepsilon^{\prime}$ with $\omega$ are sufficiently large. ${ }^{1,2)}$ However, for the PI-PS-bPM sample, $\varepsilon^{\prime}$ changes with $\omega$ very weakly (by a factor $<2 \%$ in the entire range of $\omega$ examined; cf. Figures S1-S3), which corresponds to a very small relaxation intensity and a rather weak dc conduction reflected in $\varepsilon^{\prime \prime}<<\varepsilon$. Thus, the derivative $-\{\pi / 2\} \partial \varepsilon^{\prime} / \partial \ln \omega$ could not be accurately evaluated for the PI-PS-bPM sample. For this reason, we examined the dielectric behavior of this sample through the subtracted $\varepsilon^{\prime \prime}-\sigma / \omega$.

\section{References:}

1) Wübbenhorst, M.; van Turnhout, J. Analysis of complex dielectric spectra. I. One-dimensional derivative techniques and three-dimensional modelling. J. Non-Crystalline Solids 2002, 305, 40-49.

2) Chen, Q.; Tudryn, G. J.; Colby, R. H. Ionomer dynamics and the sticky Rouse model. J. Rheol. 2013, 57, 1441-1462.

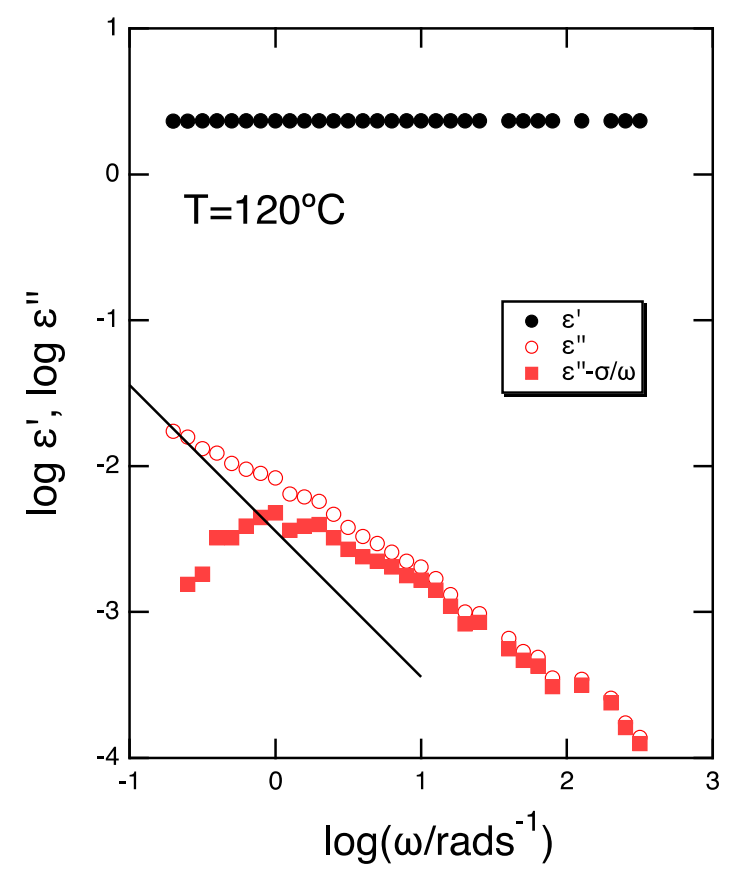

Figure S1. Dielectric data of PI-PS-bPM/A300 at $\boldsymbol{T}=\mathbf{1 2 0}^{\circ} \mathrm{C}$. 


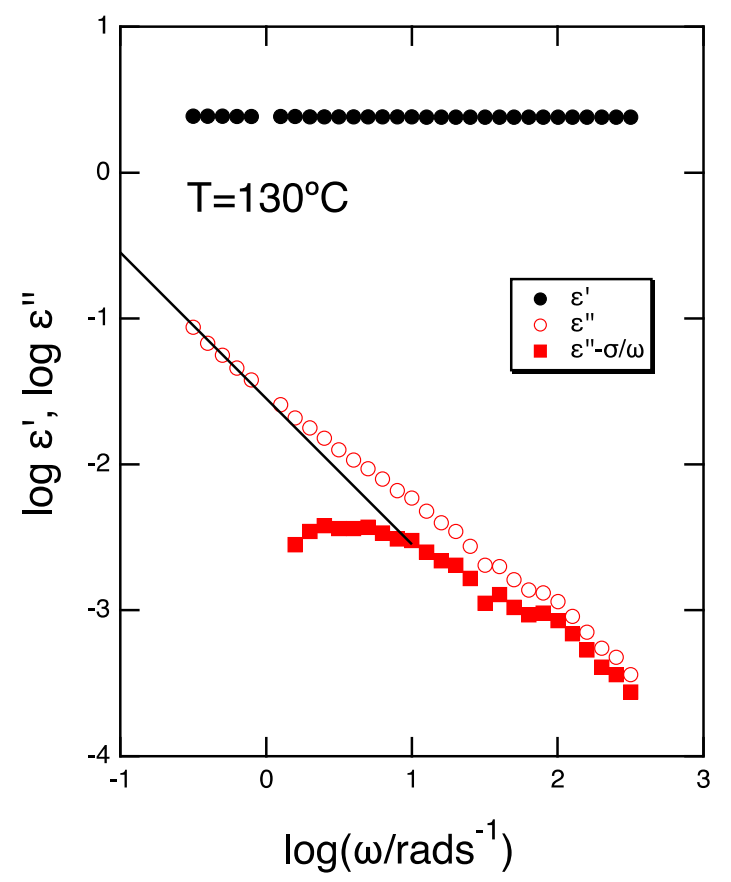

Figure S2. Dielectric data of PI-PS-bPM/A300 at $\boldsymbol{T}=\mathbf{1 3 0}^{\circ} \mathrm{C}$.

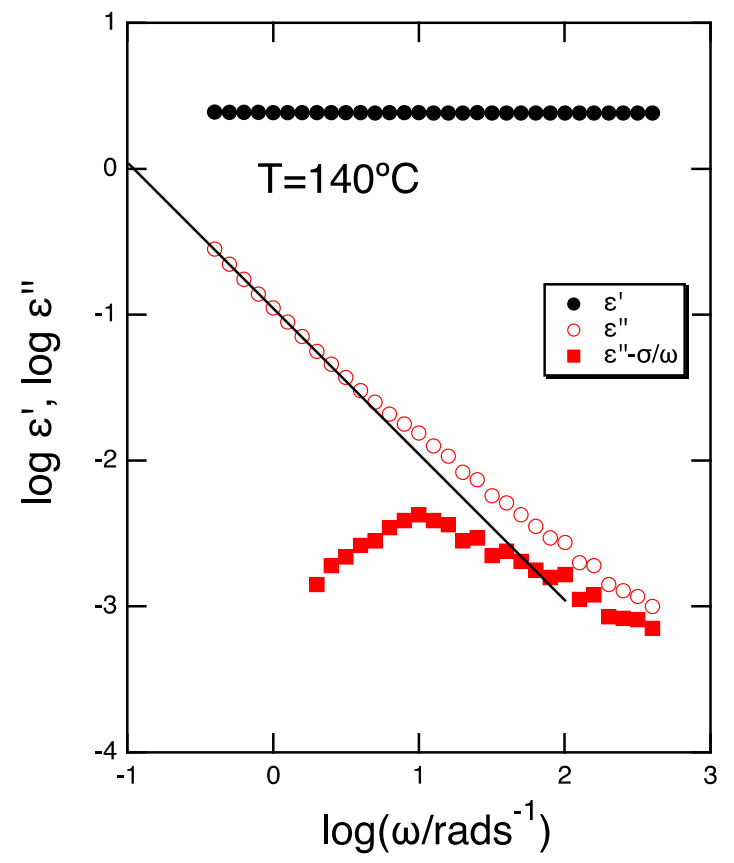

Figure S3. Dielectric data of PI-PS-bPM/A300 at $\boldsymbol{T}=\mathbf{1 4 0}^{\circ} \mathrm{C}$. 